\title{
The circadian regulation of food intake
}

\author{
Etienne Challet
}

Circadian clocks and metabolism team, Institute of Cellular and Integrative Neurosciences, Centre National de la Recherche Scientifique (CNRS), University of Strasbourg, Strasbourg, France. e-mail: challet@inci-cnrs.unistra.fr

Abstract | Feeding, which is essential for all animals, is regulated by homeostatic mechanisms. In addition, food consumption is temporally coordinated by the brain over the circadian $(\sim 24 \mathrm{~h})$ cycle. A network of circadian clocks set daily windows during which food consumption can occur. These daily windows mostly overlap with the active phase. Brain clocks that ensure the circadian control of food intake include a master light-entrainable clock in the suprachiasmatic nuclei of the hypothalamus and secondary clocks in hypothalamic and brainstem regions. Metabolic hormones, circulating nutrients and visceral neural inputs transmit rhythmic cues that permit (via close and reciprocal molecular interactions that link metabolic processes and circadian clockwork) brain and peripheral organs to be synchronized to feeding time. As a consequence of these complex interactions, growing evidence shows that chronodisruption and mistimed eating have deleterious effects on metabolic health. Conversely, eating, even eating an unbalanced diet, during the normal active phase reduces metabolic disturbances. Therefore, in addition to energy intake and dietary composition, appropriately timed meal patterns are critical to prevent circadian desynchronization and limit metabolic risks. This Review provides insight into the dual modulation of food intake by homeostatic and circadian processes, describes the mechanisms regulating feeding time and highlights the beneficial effects of correctly timed eating, as opposed to the negative metabolic consequences of mistimed eating.

\section{[H1] Introduction}

Most aspects of physiology, metabolism and behaviour display circadian rhythms (that is, endogenous rhythms close to a $24 \mathrm{~h}$ cycle), which reflects the widespread effect of circadian rhythms on biological functions. Circadian rhythmicity is generated by endogenous clocks that are highly conserved internal timing mechanisms enabling cells, organs and animals to anticipate and thereby adapt to the daily changes in their environment. More precisely, circadian clocks are intracellular mechanisms that generate self-sustained oscillations of $\sim 24 \mathrm{~h}$ by a set of specific proteins, called clock proteins, that work via autoregulatory feedback loops ${ }^{1}$. The circadian clocks produce an internal timing by the rhythmic synthesis of clockcontrolled proteins delivering intracellular and eventually extracellular temporal signals.

The endocrine glands are a remarkable example of a regulatory system whose activity is highly rhythmic on a $24 \mathrm{~h}$ cycle. In the absence of external stimulation, the daily timing of synthesis and release of hormones is highly predictable from one day to another owing to the strong influence of circadian clocks $s^{2}$. In addition to hormone synthesis and release, energy intake is predictable throughout the circadian cycle. For example, food intake is temporally organized into distinct meals that are restricted to the active phase, which is a daily period when energy stores are replenished, while the sleep phase corresponds to a daily period of fasting and mobilization of energy stores. These daily variations of energy metabolism and feeding behaviour are also coordinated by circadian clocks ${ }^{3}$.

In mammals, circadian clocks are organized in a multi-oscillatory network comprising a master clock in the suprachiasmatic nuclei (SCN) of the hypothalamus and many secondary clocks in the brain and peripheral organs, including endocrine glands, which are phase-controlled by the SCN main clock $^{4}$. Circadian rhythmicity of the SCN is generated by both neurons and astrocytes ${ }^{5-7}$.

The different circadian clocks in the body are synchronized (or reset) by cyclic environmental cues. The master clock in the SCN is mainly synchronized by ambient light detected by the retina ${ }^{8}$. A separate timing system, which in this Review is referred to as the food clock, is reset by food intake. The food clock participates in the feeding-fasting cycle and helps animals to be aroused and forage at the expected time of food availability'. A mistimed feeding time (that is, when feeding occurs during the usual resting period) can reset many circadian clocks in peripheral organs and the brain, albeit not in the $\mathrm{SCN}^{10-12}$. In addition, a mistimed feeding can have deleterious effects on metabolic health ${ }^{13,14}$. Therefore, studying the circadian regulation of food intake is important for understanding not only the basic mechanisms of energy homeostasis but also the aetiology of metabolic disorders.

This Review discusses the involvement of homeostatic and circadian processes in the daily regulation of feeding behaviour. The negative effect of mistimed eating on metabolism, which can be prevented or counteracted by timed meals during the usual active phase, is also discussed.

\section{[H1] Temporal organization of food intake}

The regulation of food intake and energy metabolism used to be mostly attributed to homeostatic feedback signals, as illustrated by the pioneering glucostatic and lipostatic theories ${ }^{15,16}$. In the past two decades, however, growing evidence shows that circadian signals have an important role in energy homeostasis ${ }^{3}$. The benefits conferred by circadian clocks are twofold. First, they provide a temporal organization from the cellular level to the organism level. Therefore, circadian clocks facilitate the temporal occurrence of related functions, such as food intake and glycogenesis, and separate conflicting functions and behaviours, such as eating and sleep. Second, circadian clocks allow organisms and organs to anticipate or be in phase with foreseeable events from one day to another, such as sunrise and sunset or food availability.

In a more simple view, the homeostatic processes that regulate food intake rely on a balance between orexigenic and anorexic factors. Orexinergic pathways gradually increase the homeostatic drive for feeding and arousal over the fasting (sleep) phase and are predominantly activated at the onset of activity ${ }^{17}$. In response to meals, anorexigenic pathways increase satiety over the active phase, which decreases the homeostatic drive for feeding to a minimum at sleep onset. In animals, evidence for circadian control in food intake is highlighted by transient hyperphagia after food deprivation. The amount of food ingested during unexpected meals markedly depends on the daily timing of food reinstatement, not only for a $24 \mathrm{~h}$ fast, but also in response to increasing duration of fasting (up to $66 \mathrm{~h}$ ). In both conditions, refed rats eat more late at night (which is at the end of their active phase) and less late in the day (which is at the end of their sleep phase) ${ }^{18}$. 
Under laboratory conditions with food and water ad libitum, rodents eat their diets in discrete feeding bouts (meals), mostly during their active phase at night. It has long been known that increased meal frequency of rats over the nocturnal phase results from a reduced postprandial satiety ${ }^{19}$. The nocturnal pattern of eating in rodents is typically bimodal, with respective peaks at dawn and dusk. Dusk meals calm hunger and facilitate replenishing of energy stores depleted in the course of sleep ${ }^{17}$. During the phase of activity onset, rats express a clear preference for carbohydrates, a selective choice of nutrients that involves the release of glucocorticoids and noradrenaline in the paraventricular nuclei (PVN) of the hypothalamus. In sharp contrast, dawn meals are aimed at providing energy during the forthcoming sleep-fasting phase. At dawn, protein and fat are preferred as macronutrients at the expense of carbohydrates ${ }^{20}$.

The eating pattern of humans is circadian in nature (meaning that it is endogenously rhythmic). The rhythmicity of human feeding was observed in a study of singly housed participants who had no external time cues (so-called free-running conditions). In these conditions, humans spontaneously have 2-3 meals during their active phase, independently of massive inter-individual variations in circadian period and duration of wakefulness ${ }^{21}$. Compared with nocturnal rodents, an oppositely phased circadian rhythm in hunger and appetite has been demonstrated in humans, with an evening peak thought to promote large meals before sleep-induced overnight fast ${ }^{22,23}$. In turn, the satiating value of ingested nutrients decreases over the waking phase ${ }^{23}$. Reminiscent of the two-process model for sleep, which states that sleep is regulated by two separate biological mechanisms ${ }^{24}$, food intake is thus regulated by both homeostatic and circadian mechanisms, which interact continuously with each other throughout the $24 \mathrm{~h}$ cycle (FIG. 1).

\section{[H1] Homeostatic control of food consumption}

Energy balance is a remarkable example of long-term homeostasis. A tight equilibrium between energy intake and expenditure and resulting body mass is kept fairly stable over time. During the fasted state, ghrelin is released from gastric cells and enters the plasma, where it transmits the major hormonal orexinergic signal to energy-sensing arcuate nuclei (ARC) of the hypothalamus, in which ghrelin stimulates release of neuropeptide $\mathrm{Y}$ (NPY) and Agouti-related peptide (AgRP) ${ }^{25}$. In turn, NPY-AgRP-expressing neurons activate second-order neurons in the lateral and perifornical hypothalamic areas, which contain orexins (hypocretins) and melanin-concentrating hormone $(\mathrm{MCH})^{26,27}$. In addition, when activated, NPY-AgRPexpressing neurons inhibit oxytocin-expressing PVN neurons to evoke acute feeding ${ }^{28}$.

The firing of orexin neurons has been directly linked to feeding behaviour ${ }^{29}$ and to energy expenditure through modulation of physical activity and the autonomic nervous system ${ }^{30}$. Excitatory inputs from the PVN, in particular, from neurons synthesizing thyrotropin-releasing hormone (TRH), feed back to AgRP neurons ${ }^{31}$. Ghrelin also provides orexinergic signals to the brainstem via the area postrema ${ }^{32}$. These coordinated effects contribute to stimulating foraging and food intake and decrease energy expenditure in rodent ${ }^{29-32}$.

After eating, the postprandial phase is marked by a number of hormonal changes that all favour satiation. Anorexigenic humoral signals are conveyed by pancreatic insulin, adipocyte-derived leptin and intestinal gluco-incretins (including glucagon-like peptide 1 (GLP1) and oxyntomodulin) to the ARC, where they converge to activate pro-opiomelanocortin (POMC) neurons ${ }^{33-35}$. $\alpha$-Melanocyte stimulating hormone ( $\alpha$-MSH) released from ARC projections binds to melanocortin 3 and 4 receptors in other hypothalamic regions, such as PVN and dorsomedial and ventromedial nuclei to mediate long-term hypophagic effects and lead to increased energy expenditure ${ }^{36,37}$.

Mice lacking Pomc expression in hypothalamic neurons are hyperphagic owing to increased meal size and duration, without modifications of meal number or daily distribution ${ }^{38}$. Histaminergic neurons of the hypothalamic tuberomammillary nuclei participate in the satiating effects of food intake, in part via the ventromedial nuclei ${ }^{39}$. Prandial hormonal cues (such as leptin, GLP1 and cholecystokinin (CCK)) also act through the area postrema on the nuclei of the solitary tract (NTS), where they activate a number of neurons, including POMC neurons, to signal acute satiation ${ }^{37,40}$. Aside from hormonal cues, visceral inputs that inform the brain of gastric distension reach the NTS via CCK-activated vagal afferents ${ }^{41}$.

The homeostatic regulation of food intake involves additional neurochemical systems, such as the endocannabinoids, that exert a widespread influence on feeding and energy metabolism. For instance, depending on the energy status - either fasted or high-fat fed - and the nature of the stimulated neurons - glutamatergic or GABAergic - activation of endocannabinoid pathways might have hyperphagic or hypophagic effects ${ }^{42}$. Their modulatory effects also vary according to the hedonic value of food, which is an important aspect of feeding that will not be covered in this Review.

In summary, the homeostatic control of hunger-linked food intake, nutrient availability and feeding cues and satiation is integrated within the brain by an intermingled network of hypothalamic and brainstem structures (FIG. 1).

\section{[H1] Circadian control of feeding}

As mentioned earlier in the Review, food intake is temporally structured across the $\sim 24 \mathrm{~h}$ circadian cycle and is strongly influenced by circadian clocks ${ }^{3}$. In general, the daily feeding-fasting cycle is altered in mice with impaired clock machinery. For instance, mice with a global deletion of clock genes, including Per1 and/or Per2, Cry1 and/or Cry2 or Rev-erba (also known as Nr1d1), display dampened day-night variations of food intake ${ }^{43-46}$. Such a behavioural defect is not always detectable under a light-dark cycle because light during daytime can inhibit food consumption in nocturnal mice, which indirectly triggers day-night (non-circadian) differences. Mice with brain-specific deletion of Rev-erba lose their circadian feedingfasting cycle, which highlights the involvement of cerebral clocks in the rhythmic behaviour of feeding ${ }^{43}$.

Variations in the concentration of MCH in the cerebrospinal fluid have been implicated in the control of feeding behaviour ${ }^{47}$. This newly identified regulation of daily behaviours might be strongly influenced by daily variations in the production rate of cerebrospinal fluid ${ }^{48}$. In humans, rates of cerebrospinal fluid production are lowest in the late afternoon and highest in late night ${ }^{49}$. Of note, the main source of cerebrospinal fluid is the choroid plexus, which houses a robust circadian $\operatorname{clock}^{50}$.

[H2] Master light-entrainable clock. It used to be a challenge to determine which brain clock, or clocks, controls the circadian rhythm of feeding. We now know that the master clock in the SCN tightly controls the sleep-wake cycle and hormonal rhythms, such as those for the release of glucocorticoids and melatonin, and participates in the daily rhythm of feeding (FIG. 2). Notably, SCN lesions lead to behavioural arrhythmicity and the loss of the feeding-fasting cycle without a change in food intake or the number of meals over a $24 \mathrm{~h}$ cycle. These data suggest that the SCN clock has a major role in the daily timing of food intake $\mathrm{e}^{51,52}$. As erratic waking states might result in an abnormal feeding pattern, it is possible that an endogenous feeding-fasting cycle is actually masked by behavioural arrhythmicity in animals with SCN lesions, which is the case for several peripheral rhythms ${ }^{53}$. 
Ambient light perceived by the retina, which itself contains a circadian clock $^{54}$, activates melanopsin-containing ganglion cells and provides photic cues that reset the SCN clock to the external light-dark cycle ${ }^{55}$. In contrast to secondary clocks, the master clock is somewhat impervious to the synchronizing effects of meal time, especially when animals are exposed to a light-dark cycle. Therefore, the master clock remains mostly entrained by light ${ }^{56,57}$. Nevertheless, the dawn peak of the bimodal pattern of nocturnal food intake in rodents could be modulated by the light-entrainable SCN $\operatorname{clock}^{17}$. In the rodent brain, the SCN projects directly into many of the hypothalamic structures that regulate food intake, and these projections probably modulate circadian control in the feeding responses ${ }^{4,58}$

[H2] The food clock - a breakfast timing system. A specific timing system, known as a food clock, tracks predictable changes in food availability and drives a rhythmic behaviour in anticipation of food availability. In animals, when access to food is limited to the resting phase, the food clock drives changes in behavioural rhythms ${ }^{9}$. In addition, some diurnal components, such as anticipatory rises in body temperature and plasma glucocorticoids, become uncoupled from the normal murine nocturnal pattern ${ }^{59}$.

An animal's response to restricted food access is best illustrated by an anticipatory bout of activity, termed food-anticipatory activity, which animals express before the expected phase of food availability ${ }^{9}$ (FIG. 2). This anticipatory bout of activity corresponds to a strong homeostatic drive for feeding. Food-anticipatory activity is mainly controlled by the food clock, which is located outside the master clock in the $\mathrm{SCN}^{9,60}$. When food is available ad libitum, the food clock, which in this state is coupled to the master clock, might de facto control the dusk peak of meals following the resting phase (in mice, this is etymologically speaking 'break-fast').

The molecular mechanisms underlying food anticipation in mammals remain controversial. The circadian properties of food-anticipatory activity have suggested that core clock genes would participate in its clock machinery (FIG. 2). Accordingly, food-anticipatory activity in mice with genetically defective clocks is markedly reduced in several studies ${ }^{61-64}$. Other researchers, however, find normal, if not increased, behavioural anticipation in the same or other strains of clock mutant mice ${ }^{65-67}$. In the latter studies, the apparent food-anticipatory activity might actually correspond to the locomotor output of a defective SCN clock that would then become sensitive to meal time. This synchronization process sometimes occurs in wild-type mice that are challenged with restricted feeding in constant darkness. In this scenario, the nocturnal pattern of activity controlled by the SCN clock becomes synchronized in anticipation to food access ${ }^{56,57}$. Further investigations in clock mutant mice with targeted SCN lesions will be needed to solve this issue. Nevertheless, altered behavioural responses in food-restricted mice with brain-specific deletion of clock genes support the notion that food-anticipatory activity is a clock-controlled rhythmic behaviour ${ }^{68,69}$. It should be noted, however, that in addition to their circadian roles, clock proteins might also have non-circadian functions that could interfere with circadian outputs, such as the feeding-fasting cycle or food-anticipatory activity. For instance, both period 2 (PER2) and REV-ERBa interact with nuclear receptors involved in numerous cellular and physiological mechanisms $^{70,71}$

A number of circadian clocks in the brain that are reset by peripheral metabolic signals might contribute to food-anticipatory rhythms controlled by the food clock. In rodents fed ad libitum with a chow diet, a daily palatable food reward can sometimes, but not always, trigger an anticipatory bout of locomotor activity and arousal ${ }^{72,73}$, suggesting that, in addition to metabolic cues, the dopaminergic reward pathways play a modulatory role in food anticipation ${ }^{74}$. As defined in this Review, food-entrainable clocks that do not drive behavioural anticipation are not considered as part of the food clock. For instance, the olfactory bulb contains a circadian clock that is reset by timed feeding, indicating that it is food-entrainable $\mathrm{e}^{75}$, but its impairment by bulbectomy or olfactory deafferentation does not prevent food-anticipatory activity in rats ${ }^{76,77}$. Despite intensive investigation, the brain location of the food clock is still elusive. The likely structures underlying the food clock might be located not only in the metabolic hypothalamus, including the ARC and lateral hypothalamus ${ }^{78-80}$, and the hindbrain (that is, the parabrachial nuclei) ${ }^{78,81}$ but also in the dorsal striatum ${ }^{82}$ and the cerebellum ${ }^{83}$ (FIG. 1).

[H2] Secondary food-entrainable clocks in the brain. Outside the master clock in the SCN, most brain regions and peripheral organs harbour secondary clocks ${ }^{84,85}$. Imposed restricted feeding in rodents has been instrumental to demonstrate that timed food intake uncouples most secondary clocks from the master clock ${ }^{10,11}$. This property was initially illustrated for circadian oscillations in peripheral tissues, such as liver, heart and lung ${ }^{10,11}$. When food access is limited to the resting phase, these clocks become entrained to meal time, while the SCN clock remains phase-locked to the lightdark cycle. Thus, in contrast to peripheral clocks, the master clock in the SCN of the hypothalamus is not shifted by timed feeding when feeding schedules are in competition with light-dark cycles ${ }^{10,11}$. In humans too, timed meals can phase-shift peripheral rhythms without affecting phase markers in the $\mathrm{SCN}^{86,87}$.

Many, but not all, cerebral clocks outside the master clock can be phase-adjusted by feeding time. This does not mean, however, that all brain food-entrainable clocks belong to the food clock and participate in the anticipation of food availability and/or feeding rhythm. Among food-entrainable clocks in the brain, the ARC contains a circadian clock that is phase-shifted by restricted feeding but remains unaltered by high-fat feeding ${ }^{12,88,89}$. In mice and rats, levels of Npy RNA in the ARC typically increase only at night ${ }^{43,90}$, while plasma concentrations of NPY are generally increased in the daytime, with peaks at dawn and dusk ${ }^{91}$. Daytime-restricted feeding leads to dampened daily expression of Npy ${ }^{90}$. RNA levels of Agrp in mice display clear daily variations with a peak in early active phase ${ }^{22,93}$. The rhythmic transcription of Agrp is probably regulated by the ARC clock, although it is noteworthy that it is also partly controlled by circulating glucocorticoids via binding of glucocorticoid response elements in the Agrp promoter ${ }^{92,93}$. While Pomc RNA levels in the ARC are consistent throughout the daily cycle, neuronal activity of $\alpha$-MSH neurons is highest in late active phase, and hypothalamic content of $\alpha$-MSH peaks at dawn $n^{43,44,58,93}$. The $\alpha$-MSH rhythm gets flattened with daytime-restricted feeding ${ }^{94}$. Hyperphagia that results from lesions to NPY receptor-expressing neurons in the ARC is associated with a loss of feeding rhythm, which highlights the importance of the ARC clock in the regulation of the daily pattern of food intake ${ }^{95}$. Furthermore, damage to AgRP and/or NPY neurons or genetic ablation of melanocortin 3 receptors reduces expression of food-anticipatory activity ${ }^{96,97}$.

Expression of $\mathrm{Hcrt}$ (which encodes orexin) and Pmch (which encodes $\mathrm{MCH}$ ), which are clock-controlled genes, displays daily variations in the lateral hypothalamus and perifornical area, with higher values at night than in the day ${ }^{43,89,90,98-100}$, while extracellular levels of orexin $\mathrm{A} \mathrm{peak} \mathrm{at} \mathrm{dawn}^{101}$. Furthermore, there are daily changes to the synaptic rearrangement of excitatory and inhibitory inputs to orexin neurons ${ }^{102}$. Oscillatory processes in the lateral hypothalamus can be phase-shifted or flattened by daytime-restricted feeding, while ablation of orexin-containing neurons severely blunts anticipation of food availability $79,90,94,103$.

The PVN are a hypothalamic hub that control neuroendocrine pathways and sympathetic outputs under circadian supervision from the SCN ${ }^{104}$. 
The PVN also harbour a weak intrinsic clock that can be phase-adjusted by meal time ${ }^{12,89}$. Neurons of the PVN that express corticotropin-releasing hormone (CRH) might participate in the circadian control of feeding because in mice impaired CRH signalling specifically increases food consumption during the resting phase, an effect that is counteracted by exogenous glucocorticoid treatment ${ }^{105}$. During a restricted feeding schedule, release of NPY in the vicinity of PVN arises from ARC, dorsomedial hypothalamic nuclei and/or NTS just before food access, concomitant with behavioural anticipation. Such a peak of NPY is thought to be controlled by the food clock because it persists during subsequent meal omission ${ }^{106}$.

The dorsomedial nuclei, which contain NPY-expressing and RFamide-related peptide 3-expressing neurons, have been involved in the control of energy balance and meal patterning at night ${ }^{107}$. In rodents, clock proteins are rhythmically expressed in the dorsomedial nuclei, but depending on the schedules of restricted feeding, these daily oscillations are more or less phase-shifted by timed feeding ${ }^{12,108}$. Regarding the role of the dorsomedial nuclei clock, it is noteworthy that dorsomedial nuclei lesions reduce the circadian amplitude of feeding rhythm and other behaviours ${ }^{109}$. Neuronal activity in the dorsomedial nuclei is slightly or strongly increased right before and during timed food access, respectively ${ }^{10,111}$. Aside from the role of the dorsomedial nuclei clock in feeding rhythm, the possible implication of the dorsomedial nuclei in behavioural anticipation of food availability has been a subject of intensive debate ${ }^{9,81}$.

In hypothalamic tuberomammillary nuclei, expression of the rate-limiting enzyme of histamine biosynthesis (histidine decarboxylase) shows daily oscillations with peak at dusk, with these oscillations being controlled by the hypothalamic tuberomammillary nuclei circadian clock ${ }^{112}$. Moreover, in rats, neuronal activity in the hypothalamic tuberomammillary nuclei is increased during the active phase under ad libitum food conditions. Although this rhythm is not phase-shifted by daytime-restricted feeding ${ }^{94}$, hypothalamic tuberomammillary nuclei neurons are selectively activated during food anticipation ${ }^{113}$. In mice that lack the histamine $\mathrm{H} 1$ receptor, the daily amount of food intake is only marginally enhanced, whereas daily feeding rhythm is blunted owing to daytime hyperphagia ${ }^{114}$. Together, these findings indicate that the neurochemical pathways of the mediobasal hypothalamus involved in the motivation to feed participate in daily feeding rhythm and the food clock.

The ventromedial nuclei display daily oscillations that are phase-shifted by daytime-restricted feeding and disrupted by genetic ablation of the clock gene Bmal1 (also known as Arntl) ${ }^{12,115}$. The ventromedial nuclei participate in increased arousal associated with food anticipation ${ }^{116}$, albeit ventromedial nuclei lesions do not markedly impair food-anticipatory activity ${ }^{117}$. Of note, without altering energy intake or its daily distribution, genetic impairment of the ventromedial nuclei clock leads to increased energy expenditure and thermogenic capacity, especially during the usual active phase ${ }^{115}$. These data, and other findings ${ }^{115}$, demonstrate that the ventromedial nuclei have a critical role in the daily control of energy expenditure via cyclic activation of brown adipose tissue thermogenic activity by the sympathetic nervous fibres ${ }^{115}$.

Circadian aspects of brainstem structures involved in food intake have been studied much less thoroughly. Daily variations in clock genes have been reported in both the NTS and parabrachial nuclei ${ }^{118,119}$. In the NTS, timing of clock gene expression is dampened in mouse models of dietinduced and genetic obesity ${ }^{118}$. However, the functional importance of these putative circadian clocks remains elusive. Rat studies have shown that in response to a timed meal, several hindbrain structures, including NTS and parabrachial nuclei, display increased neuronal activity during food access and following meals but not in anticipation ${ }^{120}$. Nevertheless, rhythmic expression of clock proteins in the parabrachial nuclei is phase-shifted by meal time, and NTS lesions impair food anticipation, indicating that this hindbrain structure could be a network link of the food clock ${ }^{81,119}$.

To sum up, the daily control of the feeding-fasting cycle relies on interactions between the master clock in the SCN, which is predominantly reset by ambient light, and secondary food-entrainable clocks, which are phase-controlled by the SCN and shifted by meal time. Several food-entrainable clocks in the brain define a food clock, that is, a clock mechanism driving rhythmic behaviours that anticipate the expected time of food availability.

\section{[H1] Keeping feeding on time}

[H2] Metabolic hormones. Various metabolic endocrine signals target not only neurons in the ARC and NTS but also brain astrocytes ${ }^{121,122}$. Among feeding-related hormones, glucocorticoids, ghrelin and glucagon secreted before meal time are recognized as pre-feeding timers. On the other hand, hormonal cues induced by meal intake or glucose production following a meal, such as insulin and leptin, can be called post-feeding timers ${ }^{123}$.

Glucorticoids, such as cortisol and corticosterone, are synthesized in the adrenal glands and released in a pulsatile manner. In the absence of stress, glucocorticoid release, which occurs in a circadian manner, is characterized by a daily increase in anticipation of the active phase ${ }^{124}$. The robust rhythmicity of glucocorticoid release is closely controlled by the SCN and acts as an internal time-giver that distributes SCN-derived circadian signals to peripheral tissues that express glucocorticoid receptors, thereby synchronizing their circadian clocks ${ }^{124}$. These secondary clocks in peripheral tissues are reset by glucocorticoids through the transcriptional modulation of clock genes, including the transient upregulation of Per1 and Per2 and downregulation of Rev-erba expression ${ }^{124}$ (FIG. 3).

When humans are fed three carbohydrate-rich meals during daytime, each meal induces an acute increase in plasma levels of cortisol that is superimposed onto the overall daily rhythm controlled by the SCN clock ${ }^{125}$. In nocturnal rats, imposed feeding during the resting phase (that is, daytime) markedly alters the temporal organization of the hypothalamo-pituitary-adrenal axis. In addition to the major rise of corticosterone at dusk, which is phased by the master clock in the SCN, a pre-prandial peak of corticosterone that occurs before food access positively modulates foodanticipatory activity ${ }^{11}$. Of note, this anticipatory peak of corticosterone is not induced by increased release of ACTH, indicating that restricted feeding cannot be considered as a classic stress ${ }^{126}$. In humans that limit their calorie intake to night-time (for example, during Ramadan), a comparable pre-prandial increase in plasma levels of cortisol is observed at dusk before the expected time for eating, while the major peak of cortisol release - known to be controlled by the SCN - is still expressed at dawn ${ }^{127}$. The anticipatory rise in glucocorticoids could have a role not only in favouring glucose supply during phases of increased energy demand but also as a time-giver to synchronize behavioural and physiological functions to coming food intake.

The pre-feeding peak of glucocorticoids might be triggered by the release of noradrenaline within the PVN ${ }^{128}$, thus suggesting that the locus coeruleus participates in the mechanisms underlying the food clock. This hypothesis is also supported by a study that shows an impaired anticipation of food availability in Ear2-deficient mice. In these mice, thelocus coeruleus, a structure critical for daily arousal, is severely impaired ${ }^{129}$. When plenty of food is available, the pre-prandial release of noradrenaline would then be phased to the onset of the active period, where it regulates carbohydrate preference, as mentioned previously ${ }^{130}$. Similarly, the pre-prandial release of corticosterone would be merged into the SCN-controlled circadian rhythm and phased to concur to anticipation of activity onset.

In humans, plasma levels of ghrelin rise before and fall after each daytime meal and progressively increase during the overnight fast phase, leading to the concept that ghrelin secretion triggers meal initiation ${ }^{131}$. In rodents, when food is provided ad libitum, plasma concentrations of ghrelin display 
a robust rhythm, which is characterized by increased levels during the resting phase. Timed food access leads to phase shifts of the circadian clock located in the stomach oxyntic cells that synthesize ghrelin and to a phase-shifted rhythm of plasma levels of ghrelin that then peaks in anticipation of food availability ${ }^{132,133}$. The relative contribution of ghrelin signalling in meal anticipation is still a subject of debate ${ }^{134}$, although impaired ghrelin receptors (including growth hormone secretagogue receptors) in both the dorsomedial nuclei and ventromedial nuclei lead to reduced behavioural anticipation to food access ${ }^{135}$.

In mice, plasma levels of glucagon, which is released by pancreatic a-cells, rise progressively over short-term food deprivation and the daily period of fasting during timed restricted feeding ${ }^{136}$. Increased levels of glucagon lead to the activation of hepatic gluconeogenesis and differential transcription of the clock genes Per1, Per 2 and Bmall via combined effects mediated by cAMP-response element-binding protein (CREB) and its co-activator CREB-regulated transcription co-activator 2 (CRTC2) ${ }^{136,137}$ (FIG. 3). The timing role of glucagon in feeding synchronization of peripheral clocks is confirmed using restricted access to diets whose ingestion does not induce insulin secretion (that is, protein-only or specific amino acids) $)^{138}$.

Meal-induced secretion of insulin from pancreatic $\beta$-cells, however, also has a key role as a post-feeding timer ${ }^{139}$. Activation of insulin signalling pathways can reset the peripheral clocks by acting at transcriptional and post-transcriptional levels. More precisely, insulin triggers hepatic transcription of Per1 and Per2, represses Rev-erba expression and stimulates AKT-mediated phosphorylation of brain and muscle ARNT-like 1 (BMAL1), which in turn suppresses its transcriptional activity ${ }^{139-141}$ (FIG. 3). Neither glucagon nor insulin signalling influences the generation of behavioural anticipation to food from one day to another ${ }^{134}$.

With respect to leptin, there is no clear evidence that its meal-induced secretion contributes to feeding synchronization, even if leptin modulates the daily rhythm of plasma levels of glucose ${ }^{123,142}$. The inhibitory role of leptin on food-anticipatory activity has been inferred from its potentiation in food-restricted rodents with impaired leptin signalling ${ }^{134}$.

Melatonin is a hormone secreted from the pineal gland only at night ${ }^{123}$. The selective nocturnal secretion delivers internal timing cues to circadian clocks ${ }^{123}$. Although not strictly speaking a metabolic hormone, melatonin can influence glucose homeostasis. Notably, human variants in the melatonin receptor MT2 are associated with an increased risk of type 2 diabetes mellitus ${ }^{143}$. Even if melatonin does affect food intake, it may indirectly modulate the regulation of feeding via its effects on circadian clocks.

Gluco-incretins, such as GLP1 and oxyntomodulin, secreted during the postprandial phase can phase-shift secondary clocks in peripheral organs $^{144,145}$. The possible role of incretins as post-feeding timers for food-entrainable clocks in the brain warrants further investigation.

Collectively, these results indicate that, among endocrine signals, glucocorticoids and pancreatic hormones are important time-givers related to food intake. Recent investigations, however, indicate that many other metabolic hormones, including adipokines and incretins, also play some role in feeding synchronization of circadian clocks. Further work is needed to unravel the proper effect of each hormone and to determine whether prefeeding and post-feeding synchronizing cues act independently from each other and whether they interact synergistically or antagonistically.

[H2] Nutrients. In addition to hormonal feeding-related cues, circulating nutrients including glucose, non-esterified fatty acids and ketone bodies probably signal to the brain to regulate the timing of cues related to peripheral energy status. Glucose supply is essential to sustain neuronal activity. However, plasma levels of glucose display daily variations that are phase-shifted by restricted feeding ${ }^{136,142}$. Changes in brain glucose availability are predominantly detected by glucose-sensing neurons located in the mediobasal hypothalamus, which includes the ARC, dorsomedial nuclei, lateral hypothalamus and ventromedial nuclei, and the NTS ${ }^{146,147}$. Aside from neurons, tanycytes, which are glucosensing glial cells of the third ventricle that contact the cerebrospinal fluid, send processes to ARC and ventromedial nuclei neighbouring regions ${ }^{148}$. In hypothalamic neurons, large changes in glucose concentration (such as from $0.5 \mathrm{nM}$ to $5.5 \mathrm{nM}$ ) affect the clock machinery by shortening the circadian period and phase-delaying Per2 $\operatorname{expression}^{149}$.

Plasma concentrations of non-esterified fatty acids increase during daily sleep and during the feeding phase in mice with a high-fat diet ${ }^{142,150}$. Lipid-sensing neurons whose firing rate is activated or inhibited by fatty acids are found in the ARC and ventromedial nuclei ${ }^{151}$. Palmitate applied on cultured mouse hypothalamic neurons affects their clock machinery by dampening expression of Per2 and Rev-erba and enhancing Clock RNA levels. Furthermore, palmitate treatment upregulates, and possibly phase-shifts, the daily expression of Npy without affecting Agrp RNA ${ }^{152}$. By contrast, high-fat feeding promotes the downregulation and flattened expression of both Npy and Agrp in the hypothalamus, while Pomc RNA levels are increased over the daily cycle compared with those during the day phase of mice on a normal diet ${ }^{150}$. Independently of diet-induced obesity, free access to a high-fat diet produces widespread changes in peripheral clocks owing to impaired CLOCK and/or BMAL1 chromatin recruitment and cyclic activation of peroxisome proliferator-activated receptor- $\gamma$ (PPAR $\gamma)$ downstream targets ${ }^{153}$. A high-fat diet available ad libitum also disrupts feeding rhythm owing to a rapid increase in daytime food consumption ${ }^{150,154}$. The underlying brain mechanisms for the high-fat diet-associated changes in feeding rhythm are not fully understood, but connexins (which are gap junction proteins) could be involved ${ }^{155}$. Of note, however, mistimed feeding is observed only in male mice and ovariectomized female mice, which indicates that ovarian hormones have a protective effect on this temporal disturbance ${ }^{156}$.

Ketone bodies, such as $\beta$-hydroxybutyrate, are released by the liver and provide an alternative energetic substrate to neurons during periods of food shortage, an effect that is counteracted by glucose supply ${ }^{136,157}$. Under high-fat feeding, hypothalamic astrocytes also convert fatty acids to ketone bodies, which might be exported to neurons to produce $\mathrm{ATP}^{158}$. Furthermore, it is meaningful that the anticipatory rise of plasma $\beta$-hydroxybutyrate before timed food access participates in the mechanisms that control food anticipation ${ }^{157}$.

[H2] Intracellular metabolic interactions. Research performed over the past 10 years has revealed functional and redundant interplays that link oscillations in the expression of clock genes and intracellular metabolism ${ }^{159}$. These data offer multiple intermingled possibilities by which feeding cues can affect the secondary circadian clocks. Transcriptional interactions are mediated by PPARs, which are transcription factors activated when bound with fatty acids that are released during either fasting or high-fat feeding. PPAR $\alpha$, which controls many target genes involved in lipid metabolism and energy homeostasis, modulates the transcription of Bmall (REF. ${ }^{160}$ ) and is a key transducer of the synchronizing effects of meal time on peripheral clocks ${ }^{136}$. PPAR $\gamma$ was mentioned earlier in the Review as a mediator of the molecular changes that occur in the circadian clock during high-fat feeding ${ }^{153}$ (FIG. 2).

The NAD ${ }^{+}$-dependent deacetylase sirtuin 1 (SIRT1) is a key intracellular metabolic sensor that directly interacts with clock proteins, CLOCK and PER2, and therefore modulates clock machinery in the SCN and peripheral clocks ${ }^{161-163}$. Poly(ADP-ribose) polymerase 1 (PARP1) is activated by 
feeding and directly fine-tunes the clock machinery by reducing the DNA-binding activity of CLOCK and/or BMAL1 (REF. ${ }^{164}$ ). In addition, AMPactivated protein kinase (AMPK) is a fuel sensor, whose activity increases in response to a reduction in the concentration of ATP within cells, which destabilizes the clock proteins cryptochrome 1 (CRY1) and PER2 (REF. ${ }^{165}$ ). The molecular clock machinery also responds to mechanistic target of rapamycin (mTOR), a crucial metabolic sensor, and therefore provides another direct metabolic modulation of the cellular clocks ${ }^{166}$ (FIG. 2).

Many clock proteins are regulated by intracellular redox status. Moreover, circadian changes in redox pathways involve daily oxidation of peroxiredoxins ${ }^{167}$. Remarkably, these oxidation rhythms result not only from interactions with the classic molecular clock machinery but also from metabolic oscillations, independent of any transcriptional mechanism, and are thought to be phylogenetically ancient clocks ${ }^{167}$ (FIG. 2).

[H2] Responsiveness of the suprachiasmatic nuclei clock to metabolic cues. Provided that a light-dark cycle is present, the SCN clock is resistant to food synchronization. Compared with secondary clocks, this difference could be because putative transducers including glucocorticoid receptor, glucagon receptors and PPARa are not expressed in the adult $\mathrm{SCN}^{124,168}$. Such resistance to food synchronization does not mean, however, that the SCN does not receive any information regarding peripheral energy status. Indeed, independently of meal timing, metabolic cues associated with calorie restriction or a high-fat diet can interfere with SCN function, whereby they alter its intrinsic period and synchronization to light ${ }^{169,170}$. Notably, SCN neurons contain glucose sensors using ATP-sensitive $\mathrm{K}^{+}$channels ${ }^{171}$. Contrary to PPAR $\alpha, \mathrm{PPAR} \beta / \delta$ is expressed in SCN cells, and its activation by fatty acids can alter SCN activity ${ }^{172}$. Cues released during fasting could be conveyed to the master clock by circulating FGF21 through $\beta$-Klotho expressed in SCN cells ${ }^{173}$. With respect to hormonal cues, modulatory effects of ghrelin and leptin on SCN responses are probably mediated via the mediobasal hypothalamus ${ }^{123,174}$, while insulin might directly inhibit the firing of SCN neurons ${ }^{123}$. Therefore, even if the master clock is not reset by meal timing under light-dark conditions, the tight crosstalk between circadian oscillations and metabolic pathways occurs in various organs, including the brain and the master clock itself.

[H2] Microbiota. Mounting evidence suggests that, depending on feeding state and diet composition, the gut microbiota influences the control of a host's energy homeostasis and modulates the development of obesity and type 2 diabetes mellitus. Microbial products, such as short-chain fatty acids and succinate, change intestinal gluconeogenesis ${ }^{175,176}$. In addition, the microbiota affects circulating signals, enteric neurons and vagal afferents that convey hunger and satiety cues to the brain ${ }^{177}$. The gut microbiota also contributes to the rhythmic interactions between gut and brain, thus participating in the circadian regulation of food intake. In particular, the gut microbiota modulates various host clocks, such as those in the colon, gut and liver, and sex-specific daily rhythms ${ }^{178-180}$. In turn, the host circadian clocks, feeding schedules and diet composition have a major effect on daily functions and oscillations of the microbiota in mice, as well as humans ${ }^{181-183}$.

In summary, the nature of the local and systemic signals that allow the synchronization of food-entrainable clocks to bouts of feeding is complex because of the multilayer implication of a wide variety of regulatory factors, from whole-organism (hormones) to intracellular pathways (enzymes and redox state) in peripheral and cerebral structures. In addition, newly identified interactions that link the gut microbiota, circadian clocks and meal time are being investigated in more detail.

\section{[H1] Negative effects of mistimed eating}

[H2] Chronodisruption. Circadian disruption and metabolic disturbances are reciprocally linked. On the one hand, the cardio-metabolic syndrome is frequently associated with alterations in the sleep-wake cycle and the circadian rhythmicity of metabolic parameters ${ }^{184}$. On the other hand, synchronizing factors that are able to reset circadian clocks, such as light, hormones and feeding time, become desynchronizers when they are perceived or provided at the wrong time of the daily cycle (for example, light at night and food intake during the usual resting phase). The miss-timing of synchronizing factors leads to circadian disturbances that, in turn, will trigger or favour adverse effects on metabolic health.

In animals, chronodisruption caused by either genetic deletion of clock genes, light at night or chronic jet-lag alters metabolic health by increasing body mass, triggering inflammation and metabolic defects and accelerating cellular ageing ${ }^{45,46,184-189}$.

In humans, epidemiological investigations highlight that circadian disruption induced by long-term shift-work is correlated with an increased risk of developing obesity and type 2 diabetes mellitus ${ }^{190,191}$. Furthermore, short-term circadian misalignment induced by forced desynchrony or acute shift in the light-dark cycle impairs glucose tolerance and reduces insulin sensitivity ${ }^{192,193}$. Importantly, the increase in diabetes mellitus risk after acute chronodisruption is distinct from the metabolic effects of sleep loss that also promote insulin resistance ${ }^{194}$.

[H2] Mistimed eating. As mentioned earlier in the Review, an imposed feeding schedule restricted to the rest phase has potent synchronizing effects on secondary clocks. In addition, mistimed eating has numerous metabolic consequences, varying according to the feeding schedule and the species. Light-fed mice with a chow diet ingest more calories and gain more body mass than night-fed controls ${ }^{14}$. Imposed fasting on mice in early night, equivalent to breakfast skipping, favours body mass gain and de novo lipid synthesis ${ }^{195}$. Light-fed rats eat less but can still gain more body mass than night-fed or ad libitum-fed animals, thus indicating increased feeding efficiency ${ }^{94}$. Moreover, spontaneous overfeeding during the rest phase is observed in chow-fed genetically obese Zucker $(f a / f a)$ rats, as is it in wild-type mice fed only with a high-fat diet ${ }^{150,196}$. During high-fat feeding ad libitum, mistimed eating takes place from the first days of fat availability, that is, before the onset of diet-induced obesity ${ }^{150}$. If food consists of only high fat limited to the resting phase (daytime), mice gain more body mass than those fed with a high-fat diet only at night ${ }^{197}$. The metabolic consequences of mistimed high-fat feeding, however, are mitigated by regular food intake during the active phase ${ }^{198}$.

In humans, skipping breakfast is associated with an increase in metabolic risk factors, including obesity and weak glycaemic control ${ }^{199,200}$. In addition, repeated late dinner in the evening or at night is significantly correlated with increased adiposity and elevated BMI ${ }^{13}$. Another study did not confirm these findings in either healthy males or females ${ }^{201}$. In addition, circadian misalignment under controlled laboratory conditions reduces insulin sensitivity ${ }^{194,202}$.

Altogether, these studies indicate that daily timing of food intake is crucial for energy balance in the short term and long term. Furthermore, both chronodisruption and mistimed eating favour increases in metabolic risks factors. 
ad libitum access to a balanced chow diet, animals fed only at night with the same diet display reduced body mass gain ${ }^{196}$. Limiting food access to the normal waking phase also improves robustness of daily oscillations at molecular (clock and metabolic genes) and physiological (respiratory exchange ratio) levels ${ }^{14}$. In mice with whole-body or liver-specific deletion of clock genes, restricting access to a chow diet prevents excessive gain in body mass and metabolic disturbances ${ }^{203}$. In humans, an increased energy intake for lunch is associated with a reduced risk of body mass gain ${ }^{204}$.

Remarkably, limiting access to an unbalanced (that is, high-fat) diet reduces diet-induced obesity and fat accumulation in the liver of mice ${ }^{205}$. In the same line, blocking spontaneous daytime overfeeding with therapeutics also prevents diet-induced obesity in mice, which further demonstrates the importance of timing food intake for energy balance ${ }^{155}$. Finally, it is noteworthy that meal timing restricted to the regular active phase prevents metabolic disturbances in animal models of shift-work ${ }^{189}$.

In short, limiting the timing of food intake - even if the ingested diet is unbalanced - to the normal active phase (that is, during the right phase) reduces metabolic disturbances.

\section{[H1] Conclusions}

The feeding-fasting cycle is controlled by a multi-oscillatory network that involves peripheral and brain clocks, including a master clock in the SCN and secondary clocks elsewhere in the body. The next challenge for researchers in the circadian field will be to determine the exact role extra-SCN brain structures within the hypothalamus and brainstem have in the regulation of the daily rhythm of feeding. Advances in circadian nutrition reveal that, aside from what and how much to eat, when to eat is also critical for energy balance. As a rule, eating during the usual active phase and combining a large breakfast and lunch with a small dinner are beneficial for metabolic health. Taking into account the timing of food intake can also be useful for improving weight loss strategies. For instance, the effectiveness of weight loss during dieting is improved in people who have their lunch around midday compared with those who eat their lunch later in the afternoon ${ }^{206}$. Similarly, weight loss in patients with severe obesity who underwent bariatric surgery is more efficient in those who have an early lunch ${ }^{207}$. On the basis of daily distribution of energy consumed, macronutrients and meal frequency, future investigations are needed to determine appropriately timed dietary patterns to prevent circadian desynchronization and limit metabolic disturbances.

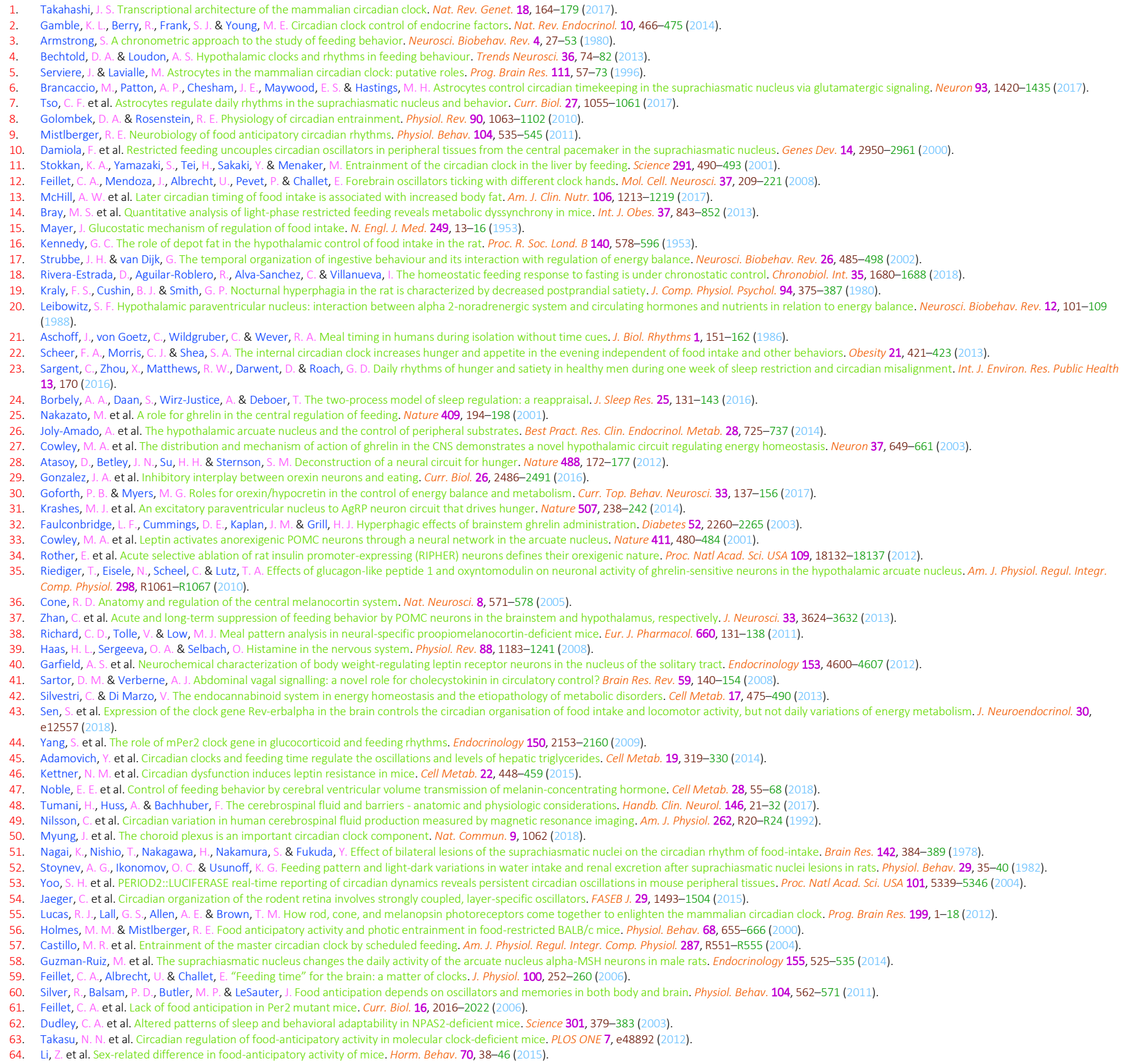


65. Pitts, S., Perone, E. \& Silver, R. Food-entrained circadian rhythms are sustained in arrhythmic Clk/Clk mutant mice. Am. J. Physiol. Regul. Integr. Comp. Physiol. 285, R57-R67 (2003).

66. Pendergast, J. S., Wendroth, R. H., Stenner, R. C., Keil, C. D. \& Yamazaki, S. mPeriod2 (Brdm1) and other single Period mutant mice have normal food anticipatory activity. Sci. Rep. 7, 15510 (2017).

67. Storch, K. F. \& Weitz, C. J. Daily rhythms of food-anticipatory behavioral activity do not require the known circadian clock. Proc. Natl Acad. Sci. USA 106, 6808-6813 (2009).

68. Mieda, M. \& Sakurai, T. Bmal1 in the nervous system is essential for normal adaptation of circadian locomotor activity and food intake to periodic feeding. J. Neurosci. 31, 15391-15396 (2011).

69. Delezie, J. et al. Rev-erbalpha in the brain is essential for circadian food entrainment. Sci. Rep. 6, 29386 (2016).

70. Duez, H. \& Staels, B. Rev-erb alpha gives a time cue to metabolism. FEBS Lett. 582, 19-25 (2008).

71. Schmutz, I., Ripperger, J. A., Baeriswyl-Aebischer, S. \& Albrecht, U. The mammalian clock component PERIOD2 coordinates circadian output by interaction with nuclear receptors. Genes Dev. 24, 345-357 (2010).

72. Mendoza, J., Angeles-Castellanos, M. \& Escobar, C. Entrainment by a palatable meal induces food-anticipatory activity and c-Fos expression in reward-related areas of the brain. Neuroscience 133, 293-303 (2005).

73. Hsu, C. T., Patton, D. F., Mistlberger, R. E. \& Steele, A. D. Palatable meal anticipation in mice. PLOS ONE 5, e12903 (2010).

74. Smit, A. N., Patton, D. F., Michalik, M., Opiol, H. \& Mistlberger, R. E. Dopaminergic regulation of circadian food anticipatory activity rhythms in the rat. PLOS ONE 8, e82381 (2013).

75. Pavlovski, I., Evans, J. A. \& Mistlberger, R. E. Feeding time entrains the olfactory bulb circadian clock in anosmic PER2::LUC mice. Neuroscience 393, 175-184 (2018).

76. Coleman, G. J. \& Hay, M. Anticipatory wheel-running in behaviorally anosmic rats. Physiol. Behav. 47, 1145-1151 (1990).

77. Davidson, A. J. et al. Food-anticipatory activity persists after olfactory bulb ablation in the rat. Physiol. Behav. 72, 231-235 (2001).

78. Blum, I. D., Lamont, E. W., Rodrigues, T. \& Abizaid, A. Isolating neural correlates of the pacemaker for food anticipation. PLOS ONE 7, e36117 (2012).

79. Mieda, M. et al. Orexin neurons function in an efferent pathway of a food-entrainable circadian oscillator in eliciting food-anticipatory activity and wakefulness. J. Neurosci. 24, 10493-10501 (2004).

80. Wiater, M. F., Li, A. J., Dinh, T. T., Jansen, H. T. \& Ritter, S. Leptin-sensitive neurons in the arcuate nucleus integrate activity and temperature circadian rhythms and anticipatory responses to food restriction. Am. J. Physiol. Regul. Integr. Comp. Physiol. 305, R949-R960 (2013)

81. Davidson, A. J. Lesion studies targeting food-anticipatory activity. Eur. J. Neurosci. 30, 1658-1664 (2009).

82. Gallardo, C. M. et al. Dopamine receptor 1 neurons in the dorsal striatum regulate food anticipatory circadian activity rhythms in mice. elife 3, e03781 (2014).

83. Mendoza, J., Pevet, P., Felder-Schmittbuhl, M. P., Bailly, Y. \& Challet, E. The cerebellum harbors a circadian oscillator involved in food antici pation. J. Neurosci. 30, 1894-1904 (2010).

84. Guilding, C. \& Piggins, H. D. Challenging the omnipotence of the suprachiasmatic timekeeper: are circadian oscillators present throughout the mammalian brain? Eur. J. Neurosci. 25, 3195-3216 (2007).

85. Albrecht, U. Timing to perfection: the biology of central and peripheral circadian clocks. Neuron $74,246-260$ (2012)

86. Krauchi, K., Cajochen, C., Werth, E. \& Wirz-Justice, A. Alteration of internal circadian phase relationships after morning versus evening carbohydrate-rich meals in humans. J. Biol. Rhythms 17, 364-376 (2002).

87. Wehrens, S. M. T. et al. Meal timing regulates the human circadian system. Curr. Biol. 27, 1768-1775 (2017).

88. Guilding, C., Hughes, A. T., Brown, T. M., Namvar, S. \& Piggins, H. D. A riot of rhythms: neuronal and glial circadian oscillators in the mediobasal hypothalamus. Mol. Brain 2, 28 (2009).

9. Abe, M. et al. Circadian rhythms in isolated brain regions. J. Neurosci. 22, 350-356 (2002).

90. Wang, D. et al. Effects of feeding time on daily rhythms of neuropeptide and clock gene expression in the rat hypothalamus. Brain Res. 1671, 93-101 (2017).

91. Jhanwar-Uniyal, M., Beck, B., Burlet, C. \& Leibowitz, S. F. Diurnal rhythm of neuropeptide Y-like immunoreactivity in the suprachiasmatic, arcuate and paraventricular nuclei and other hypothalamic sites. Brain Res. 536, 331-334 (1990).

92. Lee, B. et al. Brain-specific homeobox factor as a target selector for glucocorticoid receptor in energy balance. Mol. Cell. Biol. 33, 2650-2658 (2013).

93. Lu, X. Y. et al. Diurnal rhythm of agouti-related protein and its relation to corticosterone and food intake. Endocrinology 143, 3905-3915 (2002).

94. Ramirez-Plascencia, O. D., Saderi, N. Escobar, C. \& Salgado-Delgado, R. C. Feeding during the rest phase promotes circadian conflict in nuclei that control energy homeostasis and sleep-wake cycle in rats. Eur. Ramirez-Plascencia, O. D., Saderi, N., Escobar, C. \& Salgado-Delgado, R. C. Feeding during the rest phase promotes circadian conflict in nuclei that control energy homeostasis and sleep-wake cycle in rats. Eur. Wiater, M. F. et al. Circadian integration of sleep-wake and feeding requires NPY receptor-expressing neurons in the mediobasal hypothalamus. Am. J. Physiol. Regul. Integr. Comp. Physiol. 301, R1569-R1583 (2011).

6. Tan, K., Knight, Z. A. \& Friedman, J. M. Ablation of AgRP neurons impairs adaption to restricted feeding. Mol. Metab. 3, 694-704 (2014).

97. Girardet, C., Mavrikaki, M., Southern, M. R., Smith, R. G. \& Butler, A. A. Assessing interactions between Ghsr and Mc3r reveals a role for AgRP in the expression of food anticipatory activity in male mice. Endocrinology $155,4843-4855(2014)$

98. Fenzl, T., Flachskamm, C., Rossbauer, M., Deussing, J. M. \& Kimura, M. Circadian rhythms of basal orexin levels in the hypothalamus are not influenced by an impaired corticotropin-releasing hormone receptor type 1 system. Behav. Brain Res. 203, 143-145 (2009).

99. Feillet, C. A. et al. Rev-erbal pha modulates the hypothalamic orexinergic system to influence pleasurable feeding behaviour in mice. Addict. Biol. 22, 411-422 (2017).

100. Mahoney, C. E., Brewer, J. M. \& Bittman, E. L. Central control of circadian phase in arousal-promoting neurons. PLOS ONE 8, e67173 (2013).

101. Yoshida, Y. et al. Fluctuation of extracellular hypocretin-1 (orexin A) levels in the rat in relation to the light-dark cycle and sleep-wake activities. Eur. J. Neurosci. 14, 1075-1081 (2001).

102. Laperchia, C. et al. The excitatory/inhibitory input to orexin/hypocretin neuron soma undergoes day/night reorganization. Brain Struct. Funct. 222, 3847-3859 (2017).

103. Akiyama, M. et al. Reduced food anticipatory activity in genetically orexin (hypocretin) neuron-ablated mice. Eur. J. Neurosci. 20, 3054-3062 (2004).

104. Buijs, R. M., van Eden, C. G., Goncharuk, V. D. \& Kalsbeek, A. The biological clock tunes the organs of the body: timing by hormones and the autonomic nervous system. J. Endocrinol. 177, 17-26 (2003).

105. Muller, M. B., Keck, M. E., Zimmermann, S., Holsboer, F. \& Wurst, W. Disruption of feeding behavior in CRH receptor 1-deficient mice is dependent on glucocorticoids. Neuroreport 11, 1963-1966 (2000).

106. Yoshihara, T., Honma, S. \& Honma, K. Effects of restricted daily feeding on neuropeptide Y release in the rat paraventricular nucleus. Am. J. Physiol. 270, E589-E595 (1996).

107. Bi, S., Kim, Y. J. \& Zheng, F. Dorsomedial hypothalamic NPY and energy balance control. Neuropeptides 46, 309-314 (2012).

108. Verwey, M. \& Amir, S. Nucleus-specific effects of meal duration on daily profiles of Period1 and Period2 protein expression in rats housed under restricted feeding. Neuroscience 192, 304-311 (2011).

109. Chou, T. C. et al. Critical role of dorsomedial hypothalamic nucleus in a wide range of behavioral circadian rhythms. J. Neurosci. 23, 10691-10702 (2003).

110. Angeles-Castellanos, M., Aguilar-Roblero, R. \& Escobar, C. c-Fos expression in hypothalamic nuclei of food-entrained rats. Am. J. Physiol. Regul. Integr. Comp. Physiol. 286, R158-R165 (2004).

111. Namvar, S., Gyte, A., Denn, M., Leighton, B. \& Piggins, H. D. Dietary fat and corticosterone levels are contributing factors to meal anticipation. Am. J. Physiol. Regul. Integr. Comp. Physiol. 310, R711-R723 (2016).

112. Yu, X. et al. Circadian factor BMAL1 in histaminergic neurons regulates sleep architecture. Curr. Biol. 24, 2838-2844 (2014).

113. Meynard, M. M., Valdes, J. L., Recabarren, M., Seron-Ferre, M. \& Torrealba, F. Specific activation of histaminergic neurons during daily feeding anticipatory behavior in rats. Behav. Brain Res. 158, 311-319 (2005).

114. Masaki, T. et al. Involvement of hypothalamic histamine H1 receptor in the regulation of feeding rhythm and obesity. Diabetes 53, 2250-2260 (2004).

115. Orozco-Solis, R. et al. The circadian clock in the ventromedial hypothalamus controls cyclic energy expenditure. Cell Metab. 23, 467-478 (2016).

116. Ribeiro, A. C. et al. Two forces for arousal: Pitting hunger versus circadian influences and identifying neurons responsible for changes in behavioral arousal. Proc. Natl Acad. Sci. USA 104, 20078-20083 (2007).
117. Challet, E., Pevet, P., Lakhdar-Ghazal, N. \& Malan, A. Ventromedial nuclei of the hypothalamus are involved in the phase advance of temperature and activity rhythms in food-restricted rats fed during daytime. Brain Res. Bull. 43, 209-218 (1997).

118. Kaneko, K. et al. Obesity alters circadian expressions of molecular clock genes in the brainstem. Brain Res. 1263, 58-68 (2009).

119. Juarez, C. et al. Synchronization of PER1 protein in parabrachial nucleus in a natural model of food anticipatory activity. Eur. J. Neurosci. 35, 1458-1465 (2012).

120. Angeles-Castellanos, M., Mendoza, J., Diaz-Munoz, M. \& Escobar, C. Food entrainment modifies the c-Fos expression pattern in brain stem nuclei of rats. Am. J. Physiol. Regul. Integr. Comp. Physiol. 288, R678-R684 (2005)

121. Garcia-Caceres, C. et al. Astrocytic insulin signaling couples brain glucose uptake with nutrient availability. Cell 166, 867-880 (2016).

122. Marina, N. et al. Brain metabolic sensing and metabolic signaling at the level of an astrocyte. Glia $66,1185-1199$ (2018).

123. Challet, E. Keeping circadian time with hormones. Diabetes Obes. Metab. 17 (Suppl. 1), 76-83 (2015).

124. Oster, H. et al. The functional and clinical significance of the 24-hour rhythm of circulating glucocorticoids. Endocr. Rev. 38, 3-45 (2017).

125. Van Cauter, E., Shapiro, E. T., Tillil, H. \& Polonsky, K. S. Circadian modulation of glucose and insulin responses to meals: relationship to cortisol rhythm. Am. J. Physiol. 262, E467-E475 (1992).

126. Wilkinson, C. W., Shinsako, J. \& Dallman, M. F. Daily rhythms in adrenal responsiveness to adrenocorticotropin are determined primarily by the time of feeding in the rat. Endocrinology 104, 350-359 (1979).

127. Bogdan, A., Bouchareb, B. \& Touitou, Y. Ramadan fasting alters endocrine and neuroendocrine circadian patterns. Meal-time as a synchronizer in humans? Life Sci. 68, 1607-1615 (2001).

128. Mitome, M., Honma, S., Yoshihara, T. \& Honma, K. Prefeeding increase in paraventricular NE release is regulated by a feeding-associated rhythm in rats. Am. J. Physiol. 266, E606-E611 (1994).

129. Warnecke, M., Oster, H., Revelli, J. P., Alvarez-Bolado, G. \& Eichele, G. Abnormal development of the locus coeruleus in Ear2(Nr2f6)-deficient mice impairs the functionality of the forebrain clock and affects nociception. Genes Dev. 19, 614-625 (2005).

130. Shor-Posner, G., Azar, A. P., Jhanwar-Uniyal, M., Filart, R. \& Leibowitz, S. F. Destruction of noradrenergic innervation to the paraventricular nucleus: deficits in food intake, macronutrient selection, and compensatory eating after food deprivation. Pharmacol. Biochem. Behav. 25, 381-392 (1986).

131. Cummings, D. E. et al. A preprandial rise in plasma ghrelin levels suggests a role in meal initiation in humans. Diabetes 50, 1714-1719 (2001).

132. Bodosi, B. et al. Rhythms of ghrelin, leptin, and sleep in rats: effects of the normal diurnal cycle, restricted feeding, and sleep deprivation. Am. J. Physiol. Regul. Integr. Comp. Physiol. 287, R1071-R1079 (2004).

133. LeSauter, J., Hoque, N., Weintraub, M., Pfaff, D. W. \& Silver, R. Stomach ghrelin-secreting cells as food-entrainable circadian clocks. Proc. Natl Acad. Sci. USA 106, 13582-13587 (2009).

134. Patton, D. F. \& Mistlberger, R. E. Circadian adaptations to meal timing: neuroendocrine mechanisms. Front. Neurosci. 7, 185 (2013).

135. Merkestein, M. et al. GHS-R1a signaling in the DMH and VMH contributes to food anticipatory activity. Int. J. Obes. 38, 610-618 (2014).

136. Mukherji, A., Kobiita, A. \& Chambon, P. Shifting the feeding of mice to the rest phase creates metabolic alterations, which, on their own, shift the peripheral circadian clocks by 12 hours. Proc. Nat/ Acad. Sci. USA 112 , E6683-E6690 (2015).

137. Sun, X. et al. Glucagon-CREB/CRTC2 signaling cascade regulates hepatic BMAL1 protein. J. Biol. Chem. 290, 2189-2197 (2015).

138. Ikeda, Y. et al. Glucagon and/or IGF-1 production regulates resetting of the liver circadian clock in response to a protein or amino acid-only diet. EBioMedicine 28, 210-224 (2018).

139. Tahara, Y., Otsuka, M., Fuse, Y., Hirao, A. \& Shibata, S. Refeeding after fasting elicits insulin-dependent regulation of Per2 and Rev-erbalpha with shifts in the liver clock. J. Biol. Rhythms 26, 230-240 (2011).

140. Yamajuku, D. et al. Real-time monitoring in three-dimensional hepatocytes reveals that insulin acts as a synchronizer for liver clock. Sci. Rep. 2, 439 (2012)

141. Dang, F. et al. Insulin post-transcriptionally modulates Bmal1 protein to affect the hepatic circadian clock. Nat. Commun. 7, 12696 (2016).

142. Grosbellet, E. et al. Leptin modulates the daily rhythmicity of blood glucose. Chronobiol. Int. 32, 637-649 (2015).

143. Karamitri, A. \& Jockers, R. Melatonin in type 2 diabetes mellitus and obesity. Nat. Rev. Endocrinol. 15, 105-125 (2018).

144. Ando, H., Ushijima, K. \& Fujimura, A. Indirect effects of glucagon-like peptide-1 receptor agonist exendin-4 on the peripheral circadian clocks in mice. PLOS ONE 8, e81119 (2013).

145. Landgraf, D. et al. Oxyntomodulin regulates resetting of the liver circadian clock by food. eLife 4, e06253 (2015).
146. Mimee, A. \& Ferguson, A. V. Glycemic state regulates melanocortin, but not nesfatin-1, responsiveness of glucose-sensing neurons in the nucleus of the solitary tract. Am. J. Physiol. Regul. Integr. Comp. Physiol. 308, R690-R699 (2015).

147. Fioramonti, X., Chretien, C., Leloup, C. \& Penicaud, L. Recent advances in the cellular and molecular mechanisms of hypothalamic neuronal glucose detection. Front. Physiol. 8, 875 (2017).

148. Bolborea, M. \& Dale, N. Hypothalamic tanycytes: potential roles in the control of feeding and energy balance. Trends Neurosci. 36, 91-100 (2013).

149. Oosterman, J. E. \& Belsham, D. D. Glucose alters Per2 rhythmicity independent of AMPK, whereas AMPK inhibitor compound C causes profound repression of clock genes and AgRP in mHypoE-37 hypothalamic neurons. PLOS ONE 11, e0146969 (2016).

150. Kohsaka, A. et al. High-fat diet disrupts behavioral and molecular circadian rhythms in mice. Cell Metab. 6, 414-421 (2007).

151. Magnan, C., Levin, B. E. \& Luquet, S. Brain lipid sensing and the neural control of energy balance. Mol. Cell. Endocrinol. 418, 3-8 (2015).

152. Fick, L. J., Fick, G. H. \& Belsham, D. D. Palmitate alters the rhythmic expression of molecular clock genes and orexigenic neuropeptide Y mRNA levels within immortalized, hypothalamic neurons. Biochem. Biophys. Res.

2. Fick, L. J., Fick, G. H. \& Belsham, D. D. Palmitate alters the rhythmic expression of molecular clock genes and orexig
Commun. 413, 414-419 (2011).

153. Eckel-Mahan, K. L. et al. Reprogramming of the circadian clock by nutritional challenge. Cell 155, 1464-1478 (2013).
154. Pendergast, J. S. et al. High-fat diet acutely affects circadian organisation and eating behavior. Eur. J. Neurosci. 37, 1350

154. Pendergast, J. S. et al. High-fat diet acutely affects circadian organisation and eating behavior. Eur. J. Neurosci. 37, 1350-1356 (2013).

155. Sasaki, T. et al. A central-acting connexin inhibitor, INI-0602, prevents high-fat diet-induced feeding pattern disturbances and obesity in mice. Mol. Brain 11, 28 (2018). 


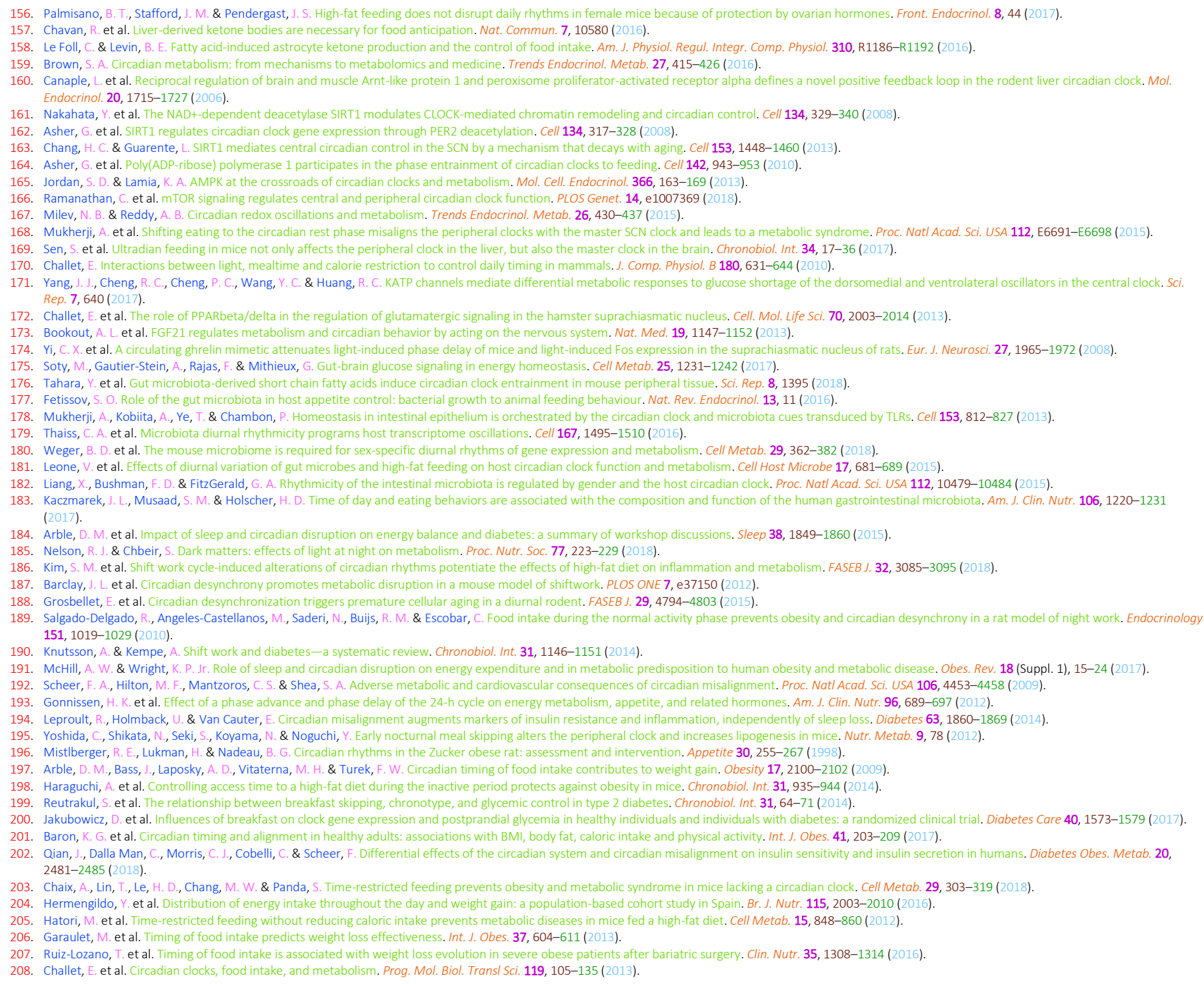

\section{Competing interests}

The author declares no competing interests.

Publisher's note

Springer Nature remains neutral with regard to jurisdictional claims in published maps and institutional affiliations.

Reviewer information

Nature Reviews Endocrinology thanks H. Piggins, R. Mistlberger and F. Scheer for their contribution to the peer review of this work.

Key points

- Short-term food consumption is regulated by a balance between orexigenic and anorexigenic factors.

- Daily pattern of eating is controlled by circadian clocks, including the master clock in the superchiasmatic nuclei reset by ambient light and other brain clocks reset by feeding time, via hormonal, nutrient and visceral cues.

- Circadian desynchronization - owing to mistimed eating or chronodisruption — has deleterious consequences on metabolic health.

- Timed dietary patterns may help to prevent circadian desynchronization and reduce metabolic disorders.

Fig. 1 | Circadian control of the daily feeding-fasting cycle by brain clocks. a | The daily feeding-fasting cycle is controlled by a multi-oscillatory system linking the light-entrainable master clock in the suprachiasmatic nuclei (SCN) of the hypothalamus with food-entrainable clocks in the metabolic hypothalamus and brainstem. In addition, animals can predict time of food availability, leading to anticipatory changes in behaviour and physiology. Such foodanticipatory changes rely on a food clock comprising food-entrainable clocks in the metabolic hypothalamus, the dorsal striatum, the cerebellum and the parabrachial nuclei (PB). Feeding and fasting cues generated by peripheral organs provide information on energy status and meal time to the brain via circulating nutrients and hormonal inputs as well as visceral neural inputs. $\mathbf{b}$ | Food intake is regulated on a daily basis by homeostatic drive (dashed grey line) and circadian gating (dotted blue line). The feeding phase at night in nocturnal rodents corresponds to a period when energy reserves are replenished, while their sleepfasting phase during daytime is a period of depletion of energy stores. ARC, arcuate nuclei; DMH, dorsomedial hypothalamic nuclei; GLP1, glucagon-like peptide 1; LH, hypothalamic lateral areas; NTS, nuclei of the solitary tract; PVN, paraventricular nuclei; TMN, tuberomammillary nuclei; VMH, ventromedial hypothalamic nuclei.

Fig. 2 | Reciprocal interactions between the circadian clocks and metabolism at cellular and systemic levels. Circadian clocks are intracellular mechanisms that generate self-sustained oscillations close to $24 \mathrm{~h}$. The molecular clock involves intermingled feedback loops of clock proteins, including CLOCK and brain and muscle ARNT-like 1 (BMAL1), that activate the transcription of many clock genes and rhythmic expression of target genes, called clock-controlled genes. In turn, PER1 and PER2, and cryptochrome 1 (CRY1) and CRY2 repress the transcriptional activity mediated by CLOCK and/or BMAL1, while REV-ERB $\alpha$, REV-ERB $\beta$ and retinoic acid receptor-related orphan receptor- $\alpha$ (ROR $\alpha)$, ROR $\beta$ and/or ROR $\gamma$ regulate the transcription of $C l o c k$ and Bmall. Clock-controlled proteins provide intracellular and extracellular rhythmic signals. Multiple functional interplays link the molecular clockwork with intracellular metabolism via changes in redox state, sirtuin 1 (SIRT1), AMP-activated protein kinase (AMPK) and other metabolic sensors and with the metabolic transcription factors called peroxisome proliferator-activated receptors (PPARs). The master clock in the suprachiasmatic nuclei (SCN), mainly reset by ambient light, adjusts the phase of secondary clocks in the brain and peripheral organs, controls the sleep-wake cycle and hormonal rhythms and participates in 
the feeding-fasting cycle. Many secondary brain clocks reset by feeding time also participate in the feeding-fasting cycle and control food-anticipatory processes. Peripheral organs, whose circadian clocks are reset by food-related signals, control the rhythmicity of glucose and lipid metabolism and generate feeding cues conveyed to the secondary clocks in the brain. Albeit not reset by timed feeding when restricted feeding schedules are in competition with light-dark cycles, the master clock in the SCN can be affected by metabolic cues associated with unbalanced diets, such as a high-fat diet or calorie restriction. mTOR, mechanistic target of rapamycin; PARP1, poly(ADP-ribose) polymerase 1. Adapted with permission from REF. ${ }^{208}$, Elsevier.

Fig. 3 | Synchronization of food-entrainable clocks by metabolic hormones. Synchronization to meal time of secondary clocks is partly mediated by pancreatic hormones and glucocorticoids. Glucagon (yellow) and glucocorticoids (blue) are pre-feeding timers because they are predominantly secreted before meal time during restricted feeding. By contrast, insulin (red) secreted in response to food intake is a post-feeding timer. It is noteworthy that both glucocorticoids and insulin signalling target the same clock genes. AKT, protein kinase B; BMAL1, brain and muscle ARNT-like 1; CLOCK, circadian locomotor output cycles kaput; CREB, cAMP-response element-binding protein; CRTC2, CREB-regulated transcriptional co-activator 2; MAPK, mitogen-activated protein kinase; PI3K, phosphoinositide 3-kinase.

\section{Glossary}

\section{Secondary clocks}

Circadian clocks found in brain structures outside of the suprachiasmatic nuclei and in peripheral organs. Self-sustained rhythmicity of extra-suprachiasmatic clocks and their cellular coupling are less robust, which might confer more flexibility to resetting cues, than the more rigid, master suprachiasmatic clock. The majority of the secondary clocks can be shifted by timed feeding (see the glossary entry for 'Food-entrainable clocks').

Free-running conditions

Housing conditions without external time cues, such as constant light or dark or constant temperature, that allow for the detection of the endogenous nature of circadian rhythms.

Clock genes

Specific genes involved in the molecular clock machinery.

Daily rhythm

A $24 \mathrm{~h}$ rhythm expressed under a light-dark cycle that is not necessarily endogenous.

Phase-shift

Change in phase of a circadian clock (or its readout, a circadian rhythm)

Food-entrainable clocks

Secondary clocks in the brain and peripheral tissues that can be phase-shifted by timed feeding.

Synchronizing factors

Sometimes called zeitgebers or time-givers; temporal signals, such as light or feeding time, that are able to reset circadian clocks, that is, to adjust their phase. 


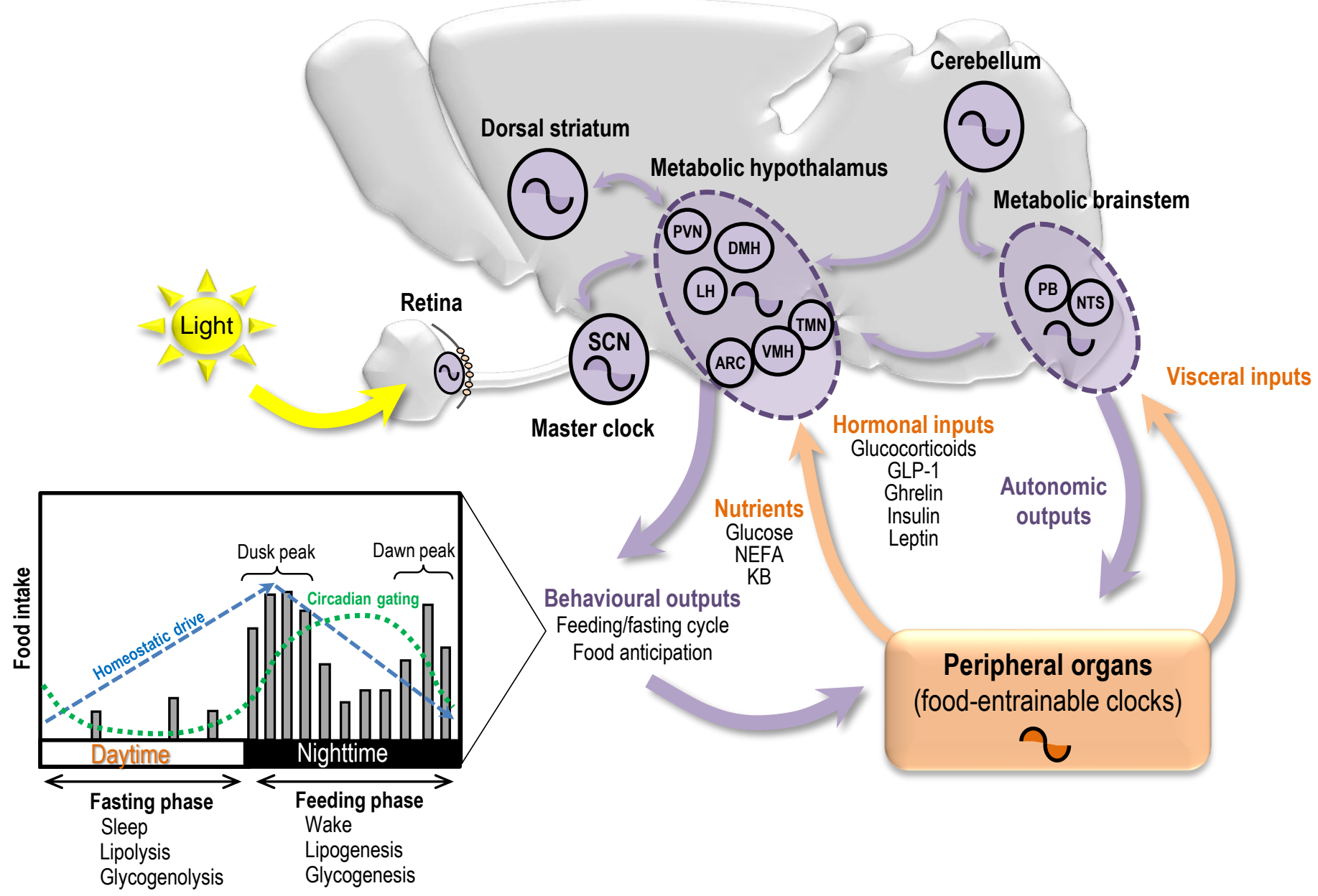

Fig. 1 revised - Challet 
Metabolic cues
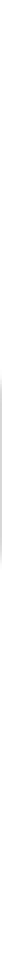

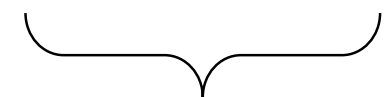

Circadian clocks 


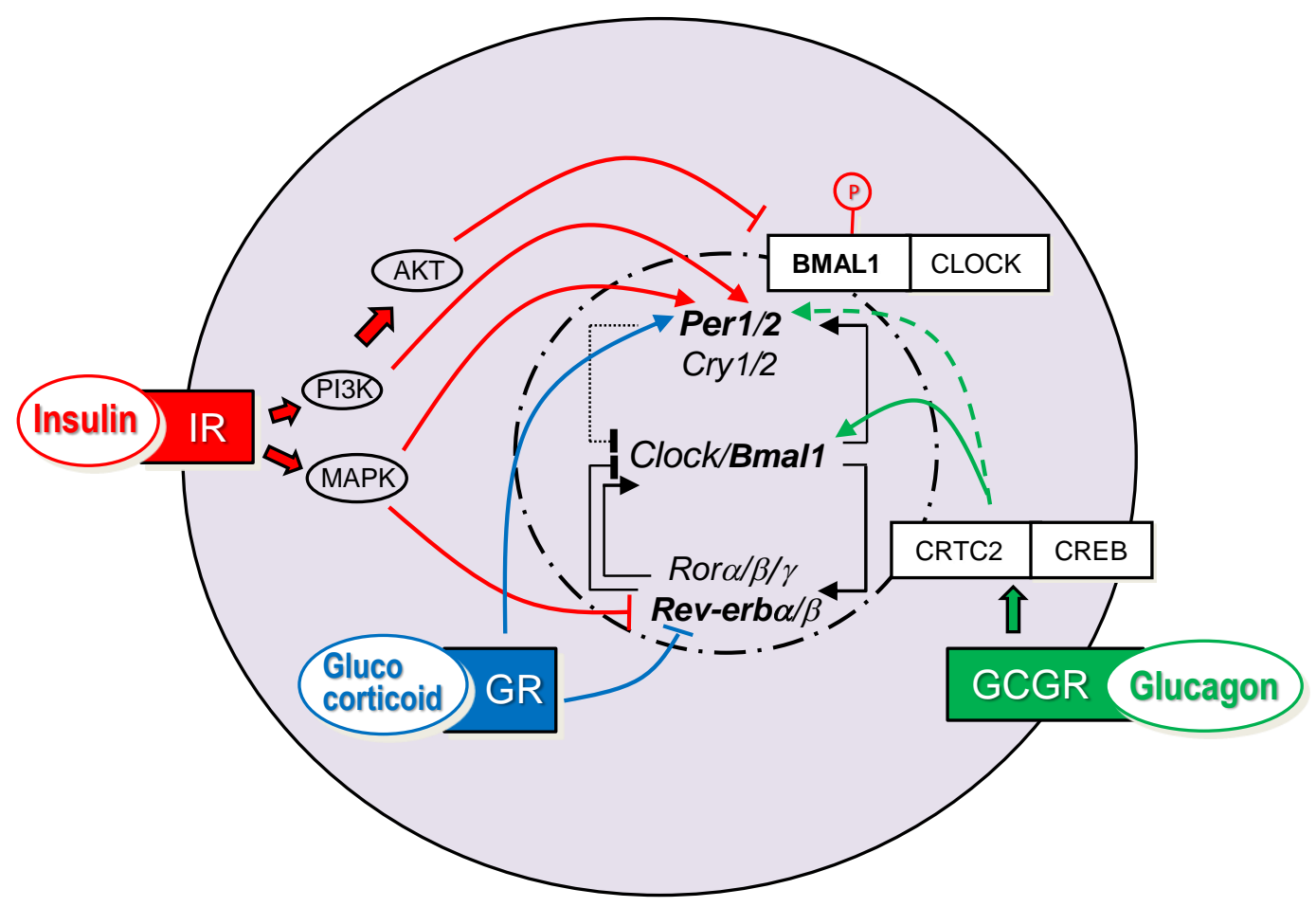

Fig. 3 revised - Challet 